Von höchstem Interesse ist die Beobachtung, daßs die Schilddrüsengeschwulst stark zurückgeht, wenn dem Teichwasser gewisse Stoffe zugefügt werden, die in der Medizin als Kropfmittel oder als Antiseptika eine Rolle spielen. Man hat experimentiert mit Jod, mit Quecksilber und mit Arsenik. Natürlich darf die Konzentration nur sehr gering sein, weil sonst die Patienten sterben würden. Man nahm höchstens 1:300 $000 \mathrm{Jod}$ als $\mathrm{KJ}, 1: 5000000$ Quecksilber als $\mathrm{HgCl}_{2}$ oder 1:300000 Arsen als $\mathrm{As}_{2} \mathrm{O}_{5}$. - Trotz dieser starken Verdünnung war deutliches Zurückgehen der Tumoren an den Versuchsfischen zu bemerken, sowohl kleine wie auch umfangreichere Geschwülste wurden günstig beeinflußt oder zum Verschwinden gebracht.

Da die Übertragung sicher nicht direkt von Fisch zu Fisch stattfindet, muB daran gedacht werden, dal3 vielleicht ein Zwischenwirt den Parasiten verbreitet. - Diese Möglichkeit liegt um so näher, als neuerdings in Südamerika beim Menschen eine Schilddrüsenkrankheit studiert worden ist, die durch eine blutsaugende Wanze verbreitet wird. - Aber auch dieser Gedanke ist einstweilen nur Hypothese.

Die prinzipielle Utbereinstimmung des Fischkropfes mit dem Kropf der Warmblüter wird dadurch bewiesen, daß es gelingt, mit Material aus einem verdächtigen Fischhälter bei Säugetieren (Ratten, jungen Hunden) Thyreoideavergrößerung hervorzubringen; man hatte den Schlamm von der Wand des Hälters abgeschabt und verfiittert. Wurde dies Material gekocht, so erwies es sich unwirksam.

Die Akten über diese hochinteressante Krankheit sind noch nicht geschlossen. Trotz aller Wahrscheinlichkeitsgründe ist der Beweis, daß ein Parasit der Erreger sei, noch nicht unwiderleglich gelungen.

Auch bleibt noch manches $\mathrm{zu}$ untersuchen bezüglich der physiologischen Wirkung der Thyreoideavergrößerung auf den Fisch. Gibt es Erscheinungen, die denen bei Basedowscher Krankheit entsprechen? - Unsere Kenntnis der normalen Physiologie der Fische ist noch nicht so ausgebildet, daß feinere Schwankungen sich nachweisen ließen; und dasselbe gilt für die Mehrzahl der Fischkrankheiten. Wahrhaft nutzbringend wird ihr Studium erst werden, wenn die Pathologie gründlichere Unterstützung von seiten der Physiologie erfährt.

\section{Die Indianer der Vereinigten Staaten von Amerika.}

Von H. Fehlinger, München.

Die Indianer im Gebiete der heutigen Vereinigten Staaten waren an Kopizahl gewis nie stark. Thre nomadische Wirtschaftsweise hatte eine geringe Bevölksrungsdichtigkeit zur Voraussetzung, und eine beträchtliche Volksvermehrung wurde durch die fortwahtrenden
Kriege der Stämme untereimander verhindert. Die Bevolkerungszahl Nordamerikas zu Beginn dex euro. päischen Kolonisation war schon oft Gegenstand der Spekulation. Gewähnlich wurde sie stark über- oder unterschätzt. Manche Extremisten bildeten sich ein, das einst Millionen von Indianern im Gebiet der Vereinigten Staaten lebten, whilend andere behaupteten, es habe trotz aller Kriege, Seuchen, Zersprengung und Zwangsansiedlung von Stämmen keine Bevölkeruugsabnahme stattgefunden. Die Vertreter dieser Ansicht stätzen sich auf Angaben der Kopfzahl, die aus verhälthismäßig neuer Zeit stammen, der Periode seit der Unabhängigkeitserklärung der Vereinigten Staaten. Es mag wohl sein, daB mit dem Ende des 18. Jahrhunderts keine bedeutende Verminderung der Volkszahl der Indianer stattfand. Vorher jedoch sind manche Stämme durch die europäischen Kolonisten ganz aufgerieben und andere arg geschwächt worden. Seuchen trugen noch bis in die dreiBiger Jahre des letzten Jahrhunderts viel zur Dezimierung der Indianer bei.

James Mooneyz) nimmt auf Grund eingehender Berechnungen an, daß zu Beginn der europäischen Besiedelung im nunmehrigen Hauptland der Vereinigten Staaten eine Indianerbevölkerung von 846000 Personen und in Alaska eine solche von 72000 Personen lebte. Im Jahre 1910 ergab die Volkszählung im Hauptand der Vereinigten Staaten 265683 und in Alaska 25331 indianische Einwohner. Zwanzig Jahre vorher wurden im Hauptlande 248253 Indianer gezählt. Doch ist zu beachten, daß unter den im Volkszählungsbericht als "Indianer" bezeichneten Personen sehr viele Mischlinge aus Ehen von Indianern mit WeiBen sind; solehe Misehehen sind häufig und im Zunehmen begriffen, so daß die Indianer als reine Rasse bald verschwunden sein werden ${ }^{2}$ ). Namentlich bei den sogen. fünf zivilisierten Stämmen im Staat Oklahoma ist die Vermischung sehr weit gediehen. Bei einem davon, dem Stamm der Cherokesen, waren seit einem Jahrhundert alle fübrendel Männer Mischlinge und 1905 waren kaum ein Viertel der Stammesangehörigen reine Indianer. Viele Stämme, namentlich jene, welche ehedem an der atlantischen Küste und am Golf von Mexiko wohnten, haben überdies einen beträchtlichen Teil Negerblut aufgenommen ${ }^{2}$ ).

In manchen Gebieten der Vereinigten Staaten haben sich nur mehr wenige Indianer erhalten; dort wurden die Indianer entweder vernichtet oder sie wurden in Reservationen in anderen Gebieten versetzt. Im Jahre $1910 \mathrm{gab}$ es in den Neu-England-Staaten, im äuBersten Nordosten der Union, bloß 2076 Indianer, in den mittelatlantischen Staaten betrug ihre Zahl 7717 , in den suidatlantischen Staaten 9054, in den südöstlichen Zentralstaaten 2612 , in den sudwestlichen Zentralstaaten 76767 (im Staate Oklahoma allein 74825 ), in den nordöstlichen Zentralstaaten 18255 , in den nordwestlichen Zentralstaaten 41406 , in den Felsengebirgsstaaten 75338 und in den Staaten an der Küste des Stillen Ozeans 32458.

Das Geschlechterverhältnis entspricht bei den Indianern fast genau jenem bei den von einheimisehen Eltern abstammenden weiBen Amerikanern; beide Bevölkerungsbestandteile weisen einen Männerüberschuß auf, während die Neger der Vereinigten Staaten einen

1) Handbook of American Indians, herausgeg. v. Bureau of Ethrology, Bd. 2, S. 287; Washington 1910.

2) Thirteenth Census of the United States, 1910, Bd. 1, S. 300. Washington 1913 .

3) Chamberlain und Mooney, Artikel "Mixed Bloods" in Handbook of. Am. Indians Bd. 1, S. 913-914. 
Franenüberschub haben. Auf je 1000 weibliche kamen männliche Personen bei den

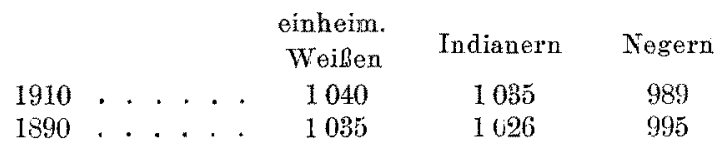

Im Jahre 1910 bestand die indianische Bevölkerung des Hauptlandes der Vereinigten Staaten aus 135133 männlichen und 130550 weiblichen Personen $(50,9$ und $49,1 \%$ ). In den meisten vorwiegend von Mischlingen bewohnten Staaten Mittel- und Südamerikas herrseht FrauentiberschuB, und dasselbe gilt von Europa mit Ausnahme der Balkanstaaten (vor den Kriegen). Männerüberschuß, wie bei den weißen und indianischen Nordamerikanern, besteht hingegen in fast allen Ländern Asiens und Afrikas, wo Volkszählungen stattfanden, ferner in allen australischen Staten. Die Ursachen dieser auffallenden Verschiedenheiten im Geschlechterverhaltnisse sind bisher nicht aufgeklärt.

Im Altersaufbau unterscheidet sich die indianiscile won- der einheimischen weiBen Bevolkerung der Vereinigten Staaten durch stärkere Besetzung der jüngeren und schwächere Besetzung der höheren Altersklassen, mit Ausnahme jener von 65 Jahren aufwärts.

Von je 1000 Personen standen im

$$
\text { Alter von }
$$

Einheim. Weiße

Indianer

weniger als 5 Jahren. . . $132 \quad 152$

5 -14 Jahren . . . . $226 \quad 256$

$15-2 \pm \quad n \quad . . . .197$

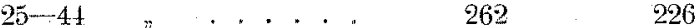

$45-64 \quad \ldots . . . .136 \quad 124$

65 oder mehr . . . . 44 49

Die verhältnismäßig größere Zahl jugendlichér Persouen wuter den Indianern ist darauf zurückzuführen, das alle Kinder aus Mischehen den Indianern zugerechnet werden, während von den Eltern aber nur eines der indianischen Bevölkerung angehört. $\mathrm{Zu}$ der Annahme einer gröberen Fruchtbarkeit der Indianer berechtigen daher die vorstehenden Zahlen nicht.

Wahrscheinlich ist allerdings bei den Indianern die Fruchtbarkeit und ebenso die Kindersterblichkeit gröBer als bei den WeiBen. Darauf weisen z. B. die Ergebnisse einer Untersuchung hin, die Dr. A. IIrdliĕka bei San-Carlos-Apachen und Pima-Indianern anstellte). Bei 37 Apachenfrauen, deren reproduktive Periode als abgeschlossen zu betrachten war, betrug die Zahl fer Geburten, ohne Fehlgeburten, insgesamt 258 oder durchschnittlieh 7 ; die höchste Kinderzahl einer Frau war 12, die geringste war 2. Auf 100 Mädchen kamen 115 Knaben. Von den Kindern überlebten zur Zeit der Erhebung $H r d l i c k a s$ aber nur noch 103 oder $40 \%, 155$ oder $60 \%$ waren gestorben. Die Sterblichkeit ist bei Knaben und Mädchen ungefithr gleich groß.

Noch ungünstiger waren die Verhältnisse bei den Pima-Indianern. Die Gesamtzahl der Kinder von 35 Pimafrauen war 246. Es trafen 112 Knaben auf je 100 Mädehen. Die Geburtenzahl der einzelnen Frauen bewegte sich zwischen 0 und 12 , im Durchschnitt betrug sie ebenfalls 7 . Von den 246 Geborenen uberlebten 83 oder $34 \%$, während 163 oder $66 \%$ gestorben waren. Von 17 Frauen beider Stämme, die je 10-12 Kinder geboren hatten, waren zusammen bloß 57 Kinder am Leben. Kinderlose When scheinen ganz selten zu sein, kinderreiche Ehen sind dagegen sehr häufig. Die Eheschliebung erfolgt im allgemeinen in einem früheren

1) Hrallička, Physiological and Medical Observations anong the Indians etc., S. 44 ff. Washington 1908.
Lebensalter als bei den Weißen; bei den noch wenig kultivierten Stämmen des Westens heiraten viele Mädchen bald nach erlangter Geschlechtsreife.

Im Jahre 1910 gestaltete sich die prozentuale Verteilung der 15 jährigen und älteren Personen nach den Zivilstand bei den Indianern und bei den einheimischen WeiRen, wie folgt:

Ledig Vexheir. Verwitw. Geschieden in Prozenten

a) Indianer :

$\begin{array}{lrrrr}\text { Männl. Geschl. } & 34,1 & 57,4 & 6,6 & 0,8 \\ \text { Weibl. Geschl. } & 21,2 & 63,8 & 13,1 & 1,2 \\ \text { Weibe: } & & & & \\ \text { Männl. Geschl. } & 38,1 & 56,3 & 4,5 & 0,5 \\ \text { Weibl. Geschl. } & 29,9 & 59,4 & 9,8 & 0,6\end{array}$

Von den Indianern ist also ein erheblich höherer Prozentsatz verheiratet oder verheiratet gewesen als von den Weißen. Auffallend ist auch die relativ große Zabl der Verwitweten und Geschiedenen unter der indianischen Bevölkerung.

Uber die Gesundheitsschädigungen der Indianer schreibt Hrdlicka in dem schon erwähnten Hand buch'), daß wenig Anhaltspunkte über jene Krankheiten vorhanden sind, die vor der Ankunft der WeiBen unter den Indianern herrschten. Die Traditionen der Indianer, ihre vielseitigen Heilriten, die zweifellos sehri alten Ursprungs sind, die heilkräftigen Eigenschaften, die vielen Pflanzenprodukten zugeschrieben werden, wie die zahlreiche Klasse der professionellen Heilkundigen, zeigen wohl an, dab Krankheiten in der vorkolumbischen Zeit nicht seltea waren. Der Zustand de $\mathrm{r}$ in alten Gräbern gefundenen Knochen, die Aussagen europäischer Reisender, die zu Anfang der Kolonialzeit mit den Indianern in Berührung kamen und der gegenwärtige Zustand mancher Stämme berechtigen jedoch zu der Annabme, daB die Indianer im ganzea eine gesunde Rasse waren. Gewisse Krankheiten der Alten Welt, wie Pocken und Rhachitis, kamen walrscheinlich in vorkolumbischer Zeit unter den Indianern nicht vor, während andere, wie Tuberkulose, Cholera, Scharlach, Krebs usw. selten waren. Seit der europäischen Kolonisation haben namentlich Tuberkusose, Syphilis und Alkoholismus unter den Indianern verheerend gewirkt. Gegenwärtig haben die Indianer, und ganz besonders die Indianermischlinge, unter vielen Krankheiten $z$ leiden. Bemerkenswert ist, dab die reinrassigen Indianer noch immer von den meisten jener schweren Krankheiten und Gebrechen frei sind, die auf defekter erblicher Veranlagung beruhen. Das weist darauf hin, daß die Blutmischung vielfach, wenn schon nicht allgemein, gegen das Lebensinteresse einer Rasse verstößt. Angeborene Mißbildungen sind sehr selten, doch ist dies darauf zuruickzufüren, rab die behafteten Kinder gewöhnlich nicht am Leben gelassen werden. Am häufigsten kommen jetzt Krankheiten der Verdauungsorgane vor; sie sind in der Kindheit die Folge ungehöriger Ernährung und namentlich des allgemeinen Genusses roher unreifer Früchte nnd $\mathrm{Ge}$. muise, im späteren Alter werden sie durch zeitweisen Nahrungsmangel, der mit ibermäBiger Nahrungsauf́nahme weehselt, sowie durch Unregelmäpigkeit der Mahlzeiten und die Vorliebe für Fett und nur halbgekochte Speisen, ubermäBigen Genub von Kaffee usw. verursacht. Andere häufige Erkrankungen sind Malaria, Bronehitis, Lungenentzuadung, Brustfellentzündung und Masern. Lungentuberkulose und Skro.

1) Handbook of Amer. Ind. I., S. 540-541. 
fulose sind häuig, besonders bei den in Reservationen in dell külteren Landesteilen wohnenden Indianem, wie z. B. bei jenen in den Staaten Dakota und Montana. Diese Indianer leben in kleinen ungesunden Hitten, die schlecht geliuftet und überfüllt sind, und die oft uberheizt werden. Die Kleidung ist schwerer als früher, die Lebensführung weniger aktiv und die Art der Nahrungsmittel hat gewechselt. Dazu kommt die völlige Unkenntnis der ansteckenden Natur der Tuberkulose. Entzündungen der Augenbindehaut treten gleichfalls häufig auf und fuhren zu Gesichtsdefekten und völhiger Blindheit. Gehörmängel werden gewöhnlich nur bei alten Leuten angetroffen. Kropf ist sehr weit verbreitet, aber selten vorherrschend; man findet ihn besonders unter einigen $Z$ weigen der Sioux, bei den Menominee, Oneida, Krähenindianern und den WeibenBerg-Apachen. Einzelne Fälle von Albinismus wurden bei einer Anzahl Stämme beobachtet; relativ häufig ist Albinismus nur bei den Hopi und den Zuñi. Frauenkrankheiten sind unter den Indianern seltener als unter den weiBen Amerikanern. Obwohl sich die Indianer Kinder wünschen und sie lieb haben, so kommt doch Kindsabtreibung bei allen Stämmen vor, die Dr. Mralička besuchte. Die alteren Leute geben die Tatsache ohne viel Zögern zu. Die Ursachen der Abtreibungen sind bei den unverheirateten Mädchen Beschämung oder Furcht, bei den verheirateten Frauen das Unvermögen, für eine große Familie zu sorgen, oder der Wunsch, den Muhen der Aufucht weiterer Kinder zu entgehen. Manchmal wird künstliche Sterilität herbeizuführen gesucht, doch sind die angewendeten Mittel wirkungslos. Getötet werden nur mißgebildete Kinder.

Von Verbrechen siad unter den Indianern Gewalttaten infolge von Trunkenheit am häufigsten. Bei normalem Geisteszustand sind die Indianer wenig zu Gewalttätigkeit, wohl aber zu Betrug und Diebstahl geneigt. Verbrechen, die mit dem Geschlechtsleben zusammenhängen, sind bei den Indianern, von Eifersuchts. delikten abgesehen, seltener als bei den weiBen $\Lambda$ merikanern. Verbrechen aus Rachsucht kommen ebenfalls selten vor.

Was die körperliche Erscheinung der Indianer betrifft, so ist vor allem $z u$ betonen, dab sie keineswegs "Rothäute" sind, noch daß sie so viele mongolische Züge aufweisen, als früher behauptet wurde. Im Gegen. teil, sie unterscheiden sich in viel mehr Merkmalen von den Mongolen, als es tbereinstimmungen swischen beiden gibt. Die Hautfarbe der Indianer ist braun in verschiedenen Schattierungen. Sehr dunkle Individuen, deren Hautfarbe Schokoladebraun nahekommt, findet man innerhalb der Vereinigten Staaten nur im fernen Sudwesten. Die dunkelsten Stellen der Haut sind, nach Hrdlick $k a^{1}$, die Handrüicken, Gelenke, der Nacken, die Achselhöhlen, die Brustwarzen, die Bauchgegend und die unbekleideten Stellen der FuRe. Die Haarfarbe ist allgemein schwarz, mit einem bläulichen oder brünnlichen Ton. Durch dauerndes Barköpfigsein erhält das Haar einen rostfarbenen Ton. Das Kopfhaar ist straff, fast kreisförmig im Durchschnitt, etwas gröber als bei den. Weißen, reich und lang. Der Bart der Männer, der meist ausgezupft wird, besteht in spärlichem Haar wuchs auf der Oberlippe und am Kinn. Der Bartwuchs scheint erheblich später aufzutreten als bei den Weißen. Auch sonst ist die Terminalbehaarung bei den Indianern spärlich entwidkelt: häufig mangelt

1) Handbook $I$, S. 53. jeder Haarwuchs sogar in den Achselhöblen und am Schamberg. Die Haut ist etwas dieker als bei den Weißen. Die Farbe der Augen bewegt sich zwischen NuBbraun und Dunkelbraun. Die Konjunktiva ist in der Jugend bläulich, bei Erwachsenen und namentlich im Alter schmutzig gelblich. Die Iris ist oft von einem schmalen aber sehr deutlich ausgeprägten Kreis umgeben.

Das Gesicht ist in der Kindheit wohl gerundet und angenehm, in der Jugend und im früheren reifen Alter interessant und gelegentlich hübseh; im Alter wird es stark runzelig. Die Kopfform, namentlich das Längenbreitenverhältnis des Kopfes, ist selbst in benachbarten Ortlichkeiten stark wechselnd. Die Stirn ist bei Erwachsenen verhältnismäßig niedrig und bei Männerm etwas nach rückwärts zurücktretend. Die Augenbranen, die vielfach ausgezupft werden, sind häufig über der Nase zusammengewachsen. Die Augenlider sind häufig herabhängend und bei Kindern ist die sog. Mongolenfalte allgemein. Die Nasenwurzel ist etwas eingesunken, und die Nase ist gewöhalich an der Basis kürzer und breiter als bei Europäern; bei Männern herrscht der aquiline Nasenrücken vor, und bei vielen reicht die Nasenspitze über die Basis des Septums herab. Die Lippen sind wohlgeformt und gewöhnlich nicht dicker als bei den Weißen. Prognathie ist dagegen stärker ausgebildet als bei der weißen Rasse. Die Ohren sind ziemlich groß und manchmal etwas dick. Der Hals ist nie lang. Der Körper ist in der Regel gut proportioniert. Die Brust ist meist genügend breit, besonders bei den Männern. Der Bauch, der bei Kindern unverhältnismäßig groß ist, behält später nur ge ringe Füile. Die Krümmung der Wirbelsäule ist mäßig, die Hiften sind relativ sehmal. Die Arme sind gut geformt und mittelmäßig muskulös, die Beinlänge ist im Verhältnis zur Gesamtlänge gewöhnlich etwas ger ringer als bei den Europäern. Die Brüste der Frauen sind von mittlerer Größe und bei kinderlosen Personen kegelförmig. Warze und Warzenhof treten stärker hervor als bei Europäerinnen. Im höheren Alter werden die Brüste klein und schlaff. Die Körpergröße ist bei den einzelnen Stämmen und innerhalb desselben Stammes sehr versebieden. Es scheint, dab sie im allgemeinen vom Nordosten nach Südwesten abrimmt, doch gibt es viele Ausnahmen von der Regel. Die individuelle Variationsbreite bewegt sich bei den meisten Stämmen und bei beiden Geschlechtern innerhalb von. $30 \mathrm{~cm}$. Die Frauen sind nach Dr. Hrdlikas Beobachtungen durchschnittlich um $121 / 2 \mathrm{~cm}$ kleiner als die Männer; der Unterschied ist bei den hochwïchsigen Stämmen größer als bei den kleinwüchsigen.

Die Indianer gehören zu den Rassen mit verhältnis mäßig geringer Differenzierung der sekundären Geschlechtsmerkmale, d. h. der Unterschied zwisehen einem erwachsenen Mann und einer erwachsenen Frau ist geringer als bei den Europäern und anderen Rassen mit weitgediehenem Geschlechtsdimorphismus. Vor allem fallt die große Ahnlichkeit der männlichen und weiblichen Gesichtsbildung auf. Die Gestalten der Frauen erinnern dureh die breite Entwicklung der Schultern und die schmalen Hüften an mänoliche Körper ${ }^{1}$. Es handelt sich dabei aber eigentlich nicht, um ein Umsehlagen des münnlichen in den weiblichen Typus, ebenso wenig als der umgekehrte Fall eintritt, sondern die Umbildung von Systemmerkmalen in Goschlechtsmerkmale ist wenig weit vorgeschritten, die

1) Vgl. Stratz, Die Rassenschönheit des Weibes, 5. Aufl., S. $74 \mathrm{ff.}$ 
Heft 52.
25. 12. 1914] Ludewig: Eine neue Methode drahtloser Telephonie. - Besprechungen.

Körperentwicklung ist, was sexuelle Differenzierung anbelangt, auf einem früheren Stadium stehen geblieben ${ }^{ \pm}$. . Der Grad der Differenzierung der System- zu Geschlechtsmerkmalen hängt jedenfalls mit der verschiedenen Schärfe der gesehlechtlichen Zuchtwahl bei den einzelnen Mensehenrassen zusammen.

\section{Eine neue Methode drahtloser Telephonie.}

Während die drahtlose Telegraphie in den letzten Jahren eine ungewöhnlich schnelle Entwicklung durchgemacht hat, ist dies bei der drahtlosen Telephonie bisher so wenig der Fall, dab man sagen kann, nachdem es gelungen war, ungedämpfte Wellen herzustellen, ist ron einem Fortschritt in diesem Spezialgebiet überbaupt nicht mehr die Rede gewesen. Allerdings sind die Erfindungen der verschiedenen Methoden zur Erzeugung von ungedämpften Schwingungen auch der drahtlosen Telephonie zugute gekommen. Sie konnte aber mit dieser Steigerung der Energie nichts anfangen, da es ibr nicht möglich war, die großen Antennenstromstärken wirksam durch die Schallschwingungen der menschlichen Sprache zu beeinflussen, da ihr ein Starkstrommikrophon fehlte.

Zur drahtlosen Telephonie waren die ersten Schwingungserzeugungsmethoden nicht zu verwenden. Diese Knallfunkenmethoden arbeiteten mit schnell abklingenden Schwingungskomplexen, die in geringer sekundlicher Aufeinanderfolge hintereinander herliefen. Man erhielt so im Telephon einen Ton, uber den naturgemäB ein Mikrophonton nicht zu ubertragen war. Erst der Paulsonsche Wasserstofflichtbogen gestattete zum ersten Male die Herstellung der ungedämpften Schwingungen, die, in der drahtlosên Telephonie verwendet, im Empfangstelephon zunächst keinen Ton erzeugen, da die Sehwingungszahl dieser Hochfrequenzströme so grob ist, dal unser Ohr sie nicht hören kann. Um sie hörbar zu machen, mußte man den Antennenstrom entweder auf der Empfangsseite periodisch unterbrechen oder auf der Sendeseite ihn in irgendeiner Weise beeinflussen, und das letztere geschah bei der drahtlosen Telephonie durch die Einschaltung eines Starkstrommikrophons. Die gewöhnlichen Mikrophone der Drahttelegraphie diirfen nur mit einem relativ geringen Strom belastet werden. Durch Parallelschaltung mehrerer Mikrophone kann man noch einen Schritt weiter kommen; aber trotzdem ist die Beeinflussung des Antennenstroms durch die sprechströme eine recht sehlechte. Man hat daher seit langem versucht - und die Anregungen dazu kommen von der Kabeltelegraphie in gleich dringender Weise wie von der drahtlosen Telephonie - ein Starkstrommikrophon zu konstruieren, also ein Mikrophon von grober Belastbarkeit. Einen Erfolg. hat bisher nur das Starkstrommikrophon von Majorana gehabt, das meist als hydraulisehes Mikrophon bezeichnet wird. Es beruht darauf, daß ein Flüssigkeitsstrahl, der aus einer AusfluBöfinung nach unten ausfließt, sich nach einer bestimmten Wegstrecke in einzelne Tropfen auflöst, und daB man die Abtrennungsstelle dieser "Tropfen dadurch leicht beeinflussen kann, daß man dea Druck an der Ausflußstelle ändert. Es werden die Schwankungen einer. Telephonmembran auf den Flüssigkeitsstrom an der Austrittsstelle übertragen. In den Strahl selbst ragen zwei Elektroden

t) Tandler-Groß, Biol. Grundlagen der sek. Geschlechtscharaktere, S. 130 . Berlin 1913. hinein. Bei Schwingungen der Telephonmembran wird so der Widerstand zwischen diesen beiden EIektroden, die den eigentlichen Mikrophonkontakt bilden, geändert.

Mit dieser Schaltung hat man bemerkenswerte Resultate erhalten. Aber auch so konnte man die starken Antennenströme, die die Erzeugung der elektrischen Wellen mittels der Hochfrequenzmaschine gezeitigt hat, nicht geniigend beeinflussen, und ein weiterer Fortschritt in der drahtlosen Telephonie war einzig durch die Konstruktion einer weiteren Verbesserung des Starkstrommikrophons zu erwarten.

Jetzt ist nun von $L$. Kiihn (Elektrotechnisehe Zeitschrift 1914, Seite 816) ein neues radiotelephonisches System beschrieben, das zwar nicht ein neues. Starkstrommikrophon verwendet, das aber trotzdem die Möglichkeit bietet, durch eine besondere Verstärkungsanordnung die Schwingungen der menschlichen. Sprache in. voller Stärke auf den Antennenstrom zur Einwirkung zu bringen. Die Anordnung beruht auf folgendem: $\mathbf{U m}$ wickelt man ein Eisenibündel mit einer Spule, so jst der Selbstinduktionskoeffizient dieser Spule von der Stärke der Magnetisierung des Eisens abhängig. Schickt man also durch eine zweitè, um den gleichen Eisenkern gewickelte Spule einen. Gleichstrom, so ist der Selbstinduktionskoeffizient der ersten Spule eine Funktion des in der Magnetisierungsspule fließenden Stromes. Es zeigt sich nuo, daß in einem bestimmten Strombereich des Magnetisierungsstromes der Selbstinduktionskoeffizient der ersten Spule sich ganz besonders gleichmäBig und schnell ändert. therlagert man nun dem Magnetisierungsstrom einen von einem. Mikrophon beeinfluBten Sprechstrom, so wird der Selbstinduktionskoeffizient der Hauptspule sich im Takte des Sprechstromes ändern. Und schaltet man weiter diese Sélbstinduktion in Serie mit einem Kondensator zu einem Schwingungskreis zusammen, so wird auch die Schwingungszahl dieses Sehwingungskreises in der gleichen Weise beeinflußt. Mit diesem Schwingungskreis ist der Antennenkreis gekoppelt und dieser wird nur dann voll erregt werden, wenn seine Schwingungszahl mit der des geschlossenen, die veränderliche Selbstinduktion enthal tenden Kreises übereinstimmt. Da sich diese Schwingungszahl aber analog den Sprechtönen ändert, so wird auch die von der Antenne ausgestrahlte Energie sich in gleieher Weise ändern. Es ist gelungen, mit einer. Energie von 8,7 Watt im. Mikrophonkreis eine Antennenenergie von 7,5 Kilowatt $z$ beeinflussen. Die neue $A n-$ ordnung, die von der Gesellschaft für drahtlose. Telegraphie durchgebildet ist, bedeutet einen wichtigen Fortschritt in der Entwicklung der drahtlosen Telephonie.

P. $L g$.

\section{Besprechungen.}

Becher, Erich, Naturphilosophie. Die Kultur der Gegenwart, ihre Entwicklung und ihre Ziele, heraus gegeben von Paul Hinneberg; dritter Teil, siebente Abteilung, erster Band, Redaktion C. Siumpf. Leipzig" und Berlin, B. G. Teubner, 1914. X, 427 S. Preis geb. M. 16,-

In Aufsützen in der Deutschen Revue habe ich mehrmals darauf hingewiesen, wie notwendig jetzt für eine Vertiefung unserer Naturanschauung auf Grund der neueren naturwissenschaftlichen wie philosophischen Errungenschaften das Zusammenarbeiten derPhilosophen und Naturforscher ist. Nachdem erst die Philosophen fär sich und dann die Naturforscher für sich die Natur- 